\title{
ON SOME APPLICATIONS OF KAKUTANI'S FIXED POINT THEOREM IN GAME THEORY
}

\author{
MD. ALAMGIR HOSSAIN ${ }^{1}$, MD. ZULFIKAR ALI'1, \\ MD. ASADUZZAMAN ${ }^{2}$ and MD. SAZZAD HOSSAIN ${ }^{3}$
}

${ }^{1}$ Department of Mathematics

University of Rajshahi

Rajshahi-6205

Bangladesh

e-mail: alamgir91rumath@gmail.com alimath1964@gmail.com

${ }^{2}$ Department of Mathematics

Islamic University

Kushtia-7003

Bangladesh

e-mail: asad@math.iu.ac.bd (CC BY 3.0).

http://creativecommons.org/licenses/by/3.0/deed.en_US

Open Access (cc) (1)


${ }^{3}$ Department of Statistics

Islamic University

Kushtia-7003

Bangladesh

e-mail: rayensl.19@gmail.com

\begin{abstract}
In this paper, we discuss some major applications of Kakutani's fixed point theorem in game theory. In the course of research work we mostly use the idea of mathematical set, functions, topological properties and Brouwer's fixed point theorem to make the Kakutani's fixed point theorem more conspicuous. In the key point of idea, we include how this theory can play the effective role to highlight new fixed point results and their applications in different fields of game theory.
\end{abstract}

\title{
1. Introduction
}

Fixed point theory has its own importance and developed tremendously for the last one and half century. In the last 50 years, fixed point theory has been an affluent field of research for many mathematicians. In the 19th century, the study of fixed point theory was initiated by Poincaré in 1886 and in 20th century developed by many mathematicians like Brouwer, Banach, Kakutani, and others. Brouwer came up with fixed point theorem to solve the equation $f(x)=x$ in 1912 . He also proved fixed point theorem for a square, a sphere and their $n$-dimensional counter parts which was further extended by Kakutani. One of the fundamental principle of functional analysis, namely-Banach Principle was introduced as well. In 1922, Banach put forward the proof that a contraction mapping in the field of a complete metric space offers a unique fixed point. It was backed up by Kannan [10]. In 1930, Schauder has given the first fixed point theorem in infinite dimensional spaces. During the first decade of last century, in 1912, the eminent Dutch mathematicians Brouwer (see [3]) exhibited a basic mathematical theorem that has seen an exceptional variety of important uses in both pure and applied mathematics. In game theory, Nash equilibrium comes under much in depth investigation. In 1928, Von Nemann \& Morgenstern could set the existence of randomized saddle point using their well- 
ON SOME APPLICATIONS OF KAKUTANI'S FIXED POINT ... 51

received minimax theorem [22] for two-person zero sum games. John Nash generalized the idea of an equilibrium to games with three or more players in his excellent work [12, 14]. Fixed point theorem is deeply associated with the existence of equilibria in games such as Brouwer's fixed point theorem [3] and the Kakutani's fixed point theorem [9]. John Von Neumann [15] established his famous minimax theorem in 1928. Later, in [21] he proposed adding his intersection lemma to it and his theory on optimal balanced growth paths in 1937. Kakutani [9] discovered a fixed point theorem in 1941 to deduce Von Neumann's minimax theorem and intersection lemma. John Nash [14] introduced his well-received equilibrium theorem in 1951. Fan [7] and Glicksberg [8] extended Kakutani's theorem in 1952 to locally convex $T_{2}$ topological vector spaces as Fan also generalized the Von Neumann intersection lemma by using his very own fixed point theorem. Fixed point theorems, more precisely Kakutani's theorem enabled proving important theorems like Nash $[13,14]$ for the case of non-cooperative games and Arrow along with Debreu [1] on general equilibrium theory.

In advanced mathematical analysis, fixed point theory is one of the most potential tools. The theorem that concerns the properties and existence of fixed points are called fixed point theorem. Informally speaking, fixed point theory is a branch of mathematics that attempts to identify all self-maps (or self-correspondence) under which at least one element is left invariant. In mathematics, a fixed point of a function is an element of the function's domain that is mapped to itself by the function. Shortly, if is a fixed point of $f(x)$ then $f(p)=p$.

Example 1.1. If $f: \mathbb{R} \rightarrow \mathbb{R}$ be defined by $f(x)=x^{2}-3 x+4$. Now, according to definition, 2 is a fixed point of the function $f$ as $f(2)=2$.

The Figure 1.1 shows the existence of three fixed points between the intersection of the function $f(x)$ and $y=x$. Consider a function $m$ which is defined as $m(p)=n(p)-p$. Then if we want to find-out the fixed point of the function $n$, then we make the solution as $m(p)=0$ (see Figure 1.2), where $f(x)=n(p)$ and $y=p($ say) in Figure 1.1). 


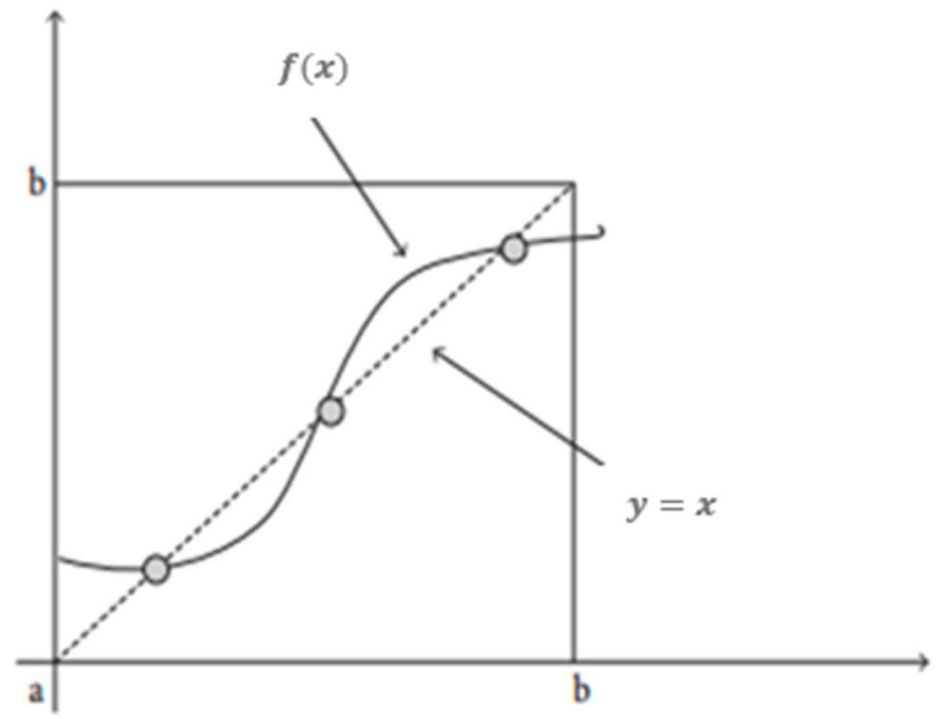

Figure 1.1. Representation of a function $f$ with three fixed points.

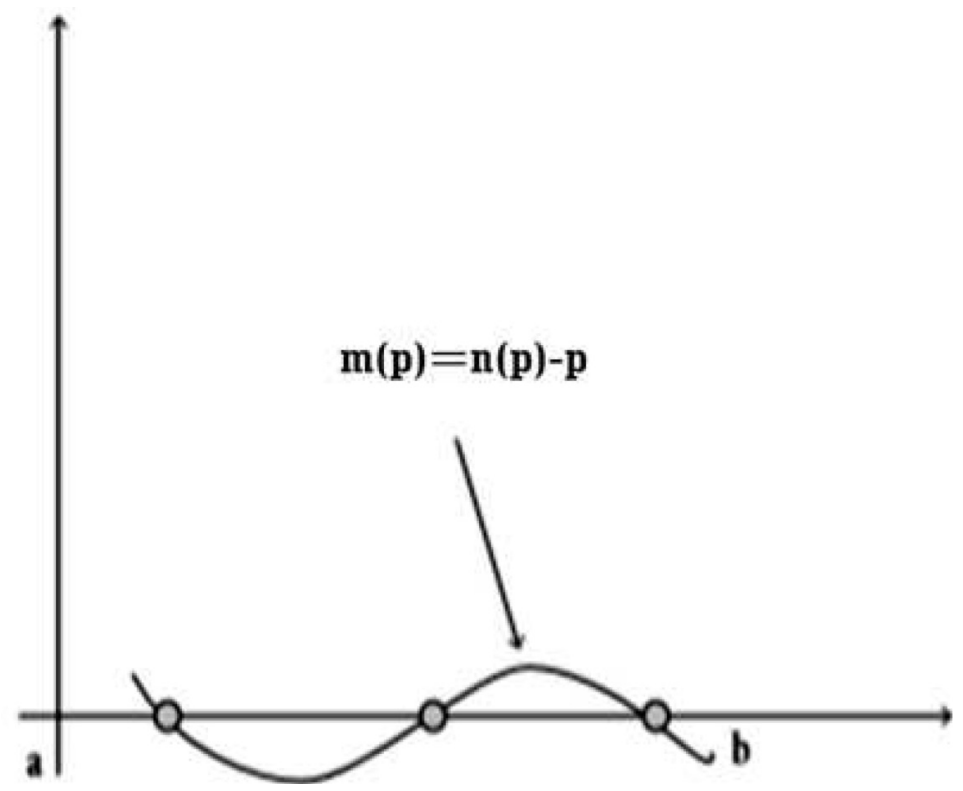

Figure 1.2. Fixed point of $n$ occurs when the solution is $m(p)=0$. 
Fixed point theory has enormous applications in different fields like mathematics, engineering, economics, game theory, image processing, artificial intelligence, population dynamics, etc. In the field of economics, it is often used in the process of determining the coincidence point of supply and demand functions. In game theory, fixed point theory is very useful because by applying this theory we can find the existence of Nash equilibrium for every finite strategic form game, for two-person zero sum games with finite strategy sets, etc. The main objective of this paper is to present some real-life applications of Kakutani's fixed point theorem in Game Theory.

\section{Preliminary Notes}

Throughout this paper, we shall use the following preliminaries which are collected from the monographs of Border [2], Buxton [4], Cobzaş [5], Eilenberg and Montgomery [6] and Scarf [18].

Definition 2.1. Let $X \neq \emptyset$. A function $F: X \rightarrow X$ is called a self map on $X$. A point $p \in X$ is called a fixed point of a self-map $F: X \rightarrow X$, if $F(p)=p$.

Example 2.1. The function $F:[0,1] \rightarrow[0,1]$ defined by $F(x)=x^{2}$ has exactly two fixed points. This function is also uniformly continuous on $[0,1]$.

Again, the function $F: \mathbb{R} \rightarrow \mathbb{R}$ defined by $F(x)=x+2$ has no fixed point in $\mathbb{R}$.

Definition 2.2. Let $(X, d)$ be a metric space. A mapping $F: X \rightarrow X$ is a contraction mapping or contraction, if there exists a constant $p$, with $0 \leq p<1$, such that $d(F(x), F(y)) \leq p d(x, y)$, for all $x, y \in X$. 
For every $x \in X$ and any $r>0$, all points $y$ in the ball $B_{r}(x)$, are mapped into a ball $B_{s}(F(x))$, with $s<r$. This is shown in Figure 2.1 as

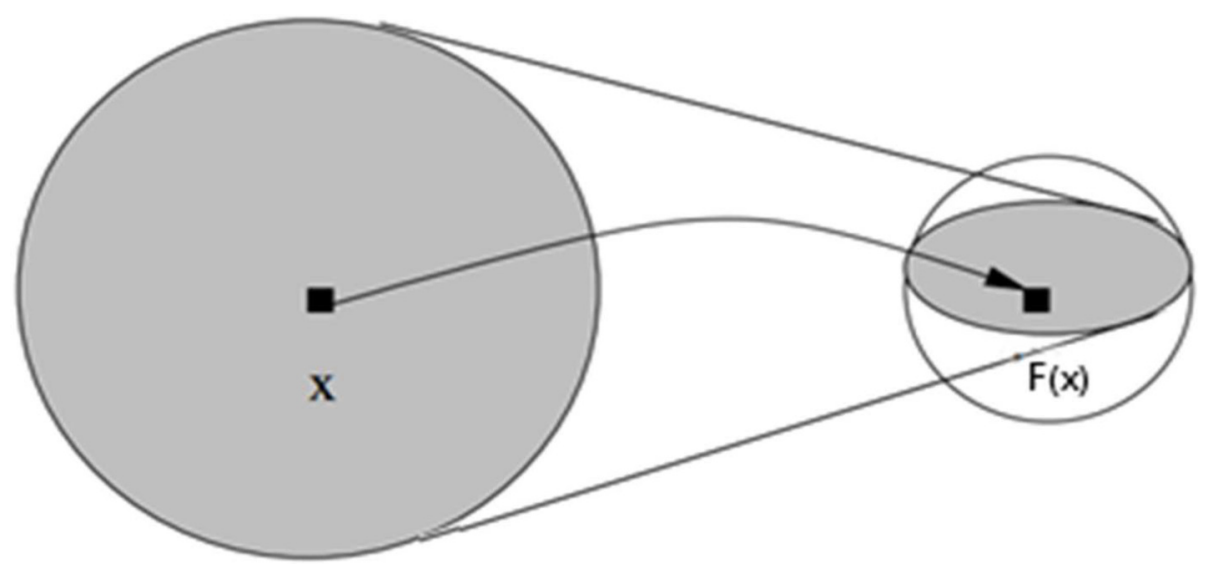

Figure 2.1. $F$ is a contraction mapping.

Sometimes a map satisfying (in Figure 2.1) with $p=1$ is called a contraction.

Example 2.2. Consider the map $f(x)=x / 2$ which maps $(0,1]$ to itself. It is clearly a contraction. If $f(x)=x$, then $x=\frac{x}{2}$ which implies $x=0$. Thus $f$ does not have a fixed point in $(0,1]$.

Theorem 2.1 (Brouwer Fixed Point Theorem in $\left.\mathbb{R}^{n}[3,4]\right)$. In the late 19th century, the idea about fixed point theory came into the mathematical concentration. To give it the mathematical sense, the mathematician Henri Poincare began using it in the topological analysis. Then in 1912 Brouwer first, published his famous fixed point theorem for finite dimension.

Statement of Brouwer fixed point theorem. This theorem states that, if $X \subseteq \mathbb{R}^{n}$ be nonempty, compact, and convex set and if $f: X \rightarrow X$ be a function which is continuous, then $f$ has a fixed point, i.e., there exist an $x \in X$ such that $f(x)=x$.

The proof of this theorem follows from [20]. 
ON SOME APPLICATIONS OF KAKUTANI'S FIXED POINT ... 55

An application of this theorem makes it possible to conclude that, a continuous function which maps from the interval $[0,1]$ to $[0,1]$ has a fixed point and we have shown it in the following figure (Figure 2.2).

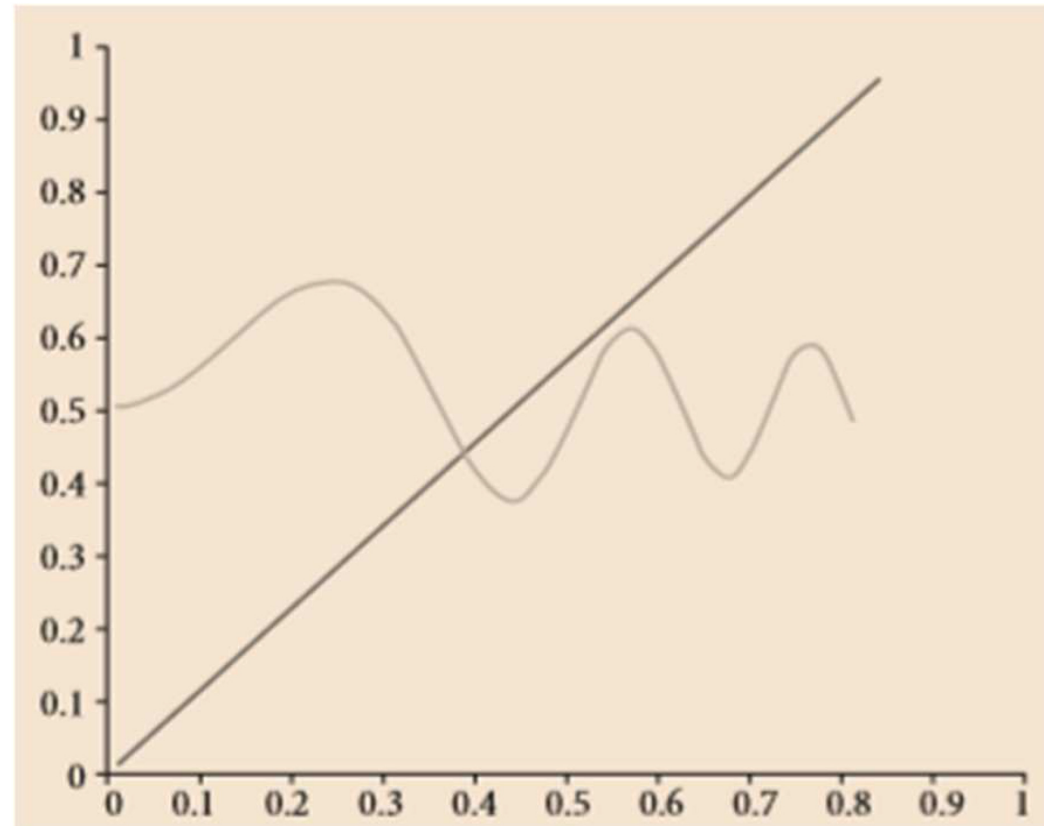

Figure 2.2. Fixed point of the continuous function within interval $[0,1]$.

In Figure 2.3 is shown a function which is not continuous so within the interval $[a, b]$ it does not have a fixed point. 


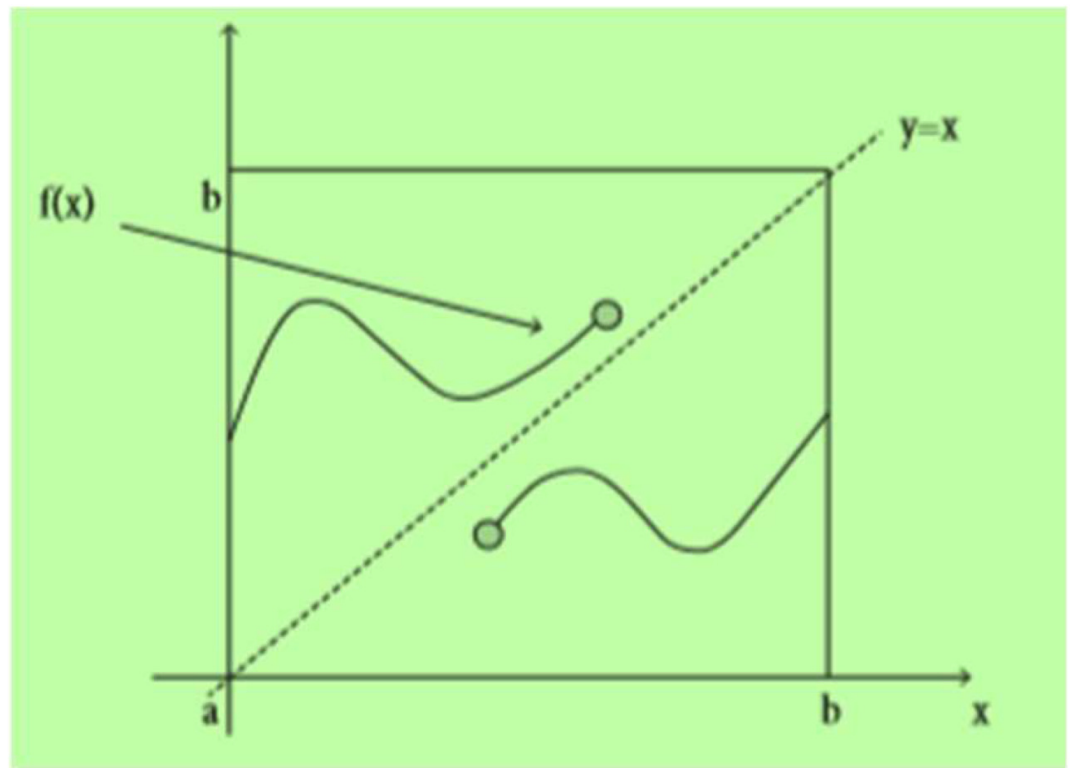

Figure 2.3. Fixed point does not exist for a function which is not continuous on $[a, b]$.

Theorem 2.2 (Kakutani's Fixed Point Theorem [3, 4]). In mathematical analysis, the Kakutani fixed-point theorem is a fixed-point theorem for setvalued functions. It provides sufficient conditions for a setvalued function defined on a convex, compact subset of a Euclidean space to have a fixed point, i.e., a point which is mapped to a set containing it. The Kakutani fixed point theorem is a generalization of Brouwer fixed point theorem.

The theorem was developed by Shizuo Kakutani [9] in 1941, and was used by John Nash in his description of Nash equilibria [12]. It has subsequently found widespread application in game theory and economics $[2]$.

Statement Kakutani's fixed point theorem. Let $X$ be a nonempty, compact and convex subset of some Euclidean space $\mathbb{R}^{n}$. Let $F: X \rightarrow 2^{X}$ be a set-valued function on $X$ with the following properties:

- $F(x)$ is non-empty and convex valued for all $x \in X$; 
- $F$ has a closed graph.

Then, $F$ has a fixed point. The proof of this theorem follows from [9].

Example. Since $x \in X$ is a fixed point of $F: X \rightarrow 2^{X}$ then $x \in f(x)$.

Let $X=(0,1)$. The function

$$
f(x)=\left\{\begin{array}{lr}
\frac{2}{3}, & 0 \leq x<0.5 \\
0.5, & x=0.5 \\
\frac{1}{4}, & 0.5 \leq x<1
\end{array}\right.
$$

satisfies all Kakutani's conditions. Then $x \in X=0.5$ is a fixed point of $f$.

\section{Applications of Kakutani's Fixed Point Theorem}

In this section, we provide some applications of Kakutani's fixed point theorem.

\subsection{Application in Nash equilibrium}

The Nash equilibrium, in game theory, according to the mathematician John Forbes Nash, is a raised solution of a game which is non-cooperative that involves two or more players in such a way that each player is considered to know about the equilibrium strategies of the other players. As a consequence, if every player changes their own strategy [17], there is no possibility to gain anything for this game.

Assume, a finite set denoted by $N$ which is fixed. This is called set of players. Now, labelling the index denoted by $j$ for every player.

Normal-form game is defined as an ordered triple in such a way that, $v_{j}$ be the functions $v_{j}: \prod_{j \in N} S_{j} \rightarrow \mathbb{R}$, where $S_{j}$ is non-empty sets for all $j \in N$. Then obviously, we consider $S_{j}$ and $j$ as a set of strategies, 
and as a user's gain (utility) function $(j \in N)$ respectively. If we denote $S_{N}=\prod_{j \in N} S_{j}$, then every, $s \in S_{N}$ is the outcome (strategy profile) in the game $\Gamma$. Player $j$ choose strategy, $s_{j} \in S_{j}$. The outcome of the game $s$ and gain for every player $j-v_{j}(s)$ is chosen only when all the players select their strategies. By the way of defining the three elements are given below, we can tell then the single-form of game is possible to define from the aforesaid discussion:

(i) Set of game for players.

(ii) For each player, there is a set of strategy.

(iii) For each player, there is a gain function.

At first, we will be inaugurated to various suitable notations.

Let, $s=\left(s_{1}, s_{2}, \ldots, s_{n}\right)$ be a strategic profile. Then:

(1) $s_{-j}=\left(s_{1}, s_{2}, \ldots, s_{j-1}, s_{j+1} \ldots, s_{n}\right)$;

(2) $\left(s_{-j}, s_{j}^{*}\right)=\left(s_{1}, s_{2}, \ldots, s_{j-1}, s_{j}^{*}, s_{j+1}, \ldots, s_{n}\right)$.

Therefore, Nash equilibrium is defined to be the strategic sketch $s^{*} \in S$ in such a way that for each $j \in N$ remains that $v_{j}\left(s_{-j}^{*}, s_{j}^{*}\right) \geq v_{j}\left(s_{-j}^{*}, s_{j}\right)$ for, $s_{j} \in S_{j}$.

Theorem 3.1.1 (Nash theorem [13, 14]). If strategic sets of each player follow the following criteria:

(i) Non-empty, convex and compact.

(ii) Their utility functions are continuous and quasi-concave.

Then, for the case of a normal form game in the type, $s_{-j}$, Nash equilibrium exists.

Now, by applying the theorem of Kakutani's fixed point, we can define $p_{j}\left(s_{-j}\right)=\arg \max \left\{v_{j}\left(s_{j}, s_{-j}\right): s_{j} \in S_{j}\right\}$ and $p(s)=\prod_{j=1}^{n} p_{j}\left(s_{-j}\right)$. On the basis of Weierstrass theorem function $p$ is well defined (which states 
that, let $X \subset \mathbb{R}^{n}$ and let $f: X \rightarrow \mathbb{R}$ be a continuous function on $X$. If $X$ is compact, then $f$ attains a maximum and a minimum on $X$ ). It should be noticed that if $s^{*} \in p\left(s^{*}\right)$ then, $s_{j}^{*} \in p\left(s_{-j}^{*}\right)$ for each $j \in N$, which makes the assumption that $s^{*}$ is Nash equilibrium.

Lemma 3.1.1. If $X_{1}$ and $X_{2}$ are compact Hausdroff topological space and $P: X_{1} \times X_{2} \rightarrow \mathbb{R}$ is continuous, then the functions $f(x)=\min$ $p\left(x, X_{2}\right), x \in X_{1} ; g(x)=\max P\left(X_{1}, y\right), y \in X_{2}$ are continuous too.

Proposition 3.1.1. Let $X, Y$ be two topological spaces and $T: X \rightarrow Y$ be a set-valued mapping.

(1) If $Y$ is regular, $T$ is upper semi continuous and for every $a \in X$ the set $T(a)$ is nonempty and closed, then $T$ has a closed graph.

(2) Conversely, if the space $Y$ is compact Hausdroff and $T$ is with closed graph, then $T$ is upper semi continuous.

Proposition 3.1.2. If $P_{x}=\left\{y \in X_{2}: T(x, y)=f(x)\right\}$ and $Q_{y}=\{x \in$ $\left.X_{1}: T(x, y)=g(y)\right\}, \forall x \in X_{1}$ and $y \in X_{2}$, then $P_{x}$ and $Q_{y}$ are nonempty and closed for all $(x, y) \in X_{1} \times X_{2}$.

Theorem 3.1.2. Let $X_{1}$ and $X_{2}$ are compact Hasudroff topological space and $X=X_{1} \times X_{2}$. Again, we define $T: X \rightarrow X$ by $T(x, y)=P_{y} \times Q_{x}$, for all, $(x, y) \in X, T(x, y)$ is a nonempty convex subset of $X,\{((x, y), T(x, y))\}$ is closed. Then there exist $x^{*}=\left(x_{0}, y_{0}\right)$ such that $x^{*} \in T\left(x^{*}\right)$.

Proof: Assume that, $X_{1}$ and $X_{2}$ are compact Hasudroff topological space and also $X=X_{1} \times X_{2}$. Again, we define $T: X \rightarrow X$ by $T(x, y)=P_{y} \times Q_{x}$ for all $(x, y) \in X$. 
Since $P_{y}$ and $Q_{x}$ are non-empty and closed sets for every $(x, y) \in X_{1} \times X_{2}$, it follows that $T(x, y)$ is non-empty and closed subset of $X$.

Consider $G_{T}=\{((x, y), T(x, y)) \in X \times X\}$ be a graph for $T$.

Since, $\{((x, y), T(x, y)\}$ is closed, then according to definition it follows that $T$ has a closed graph.

Now, by the Proposition 3.1.1 since $T$ is upper semi continuous, so that by (Kakutani's fixed point theorem in locally convex case [23]), $T$ has a fixed point $x^{*}=\left(x_{0}, y_{0}\right)$.

That is $\left(x_{0}, y_{0}\right) \in T\left(\left(x_{0}, y_{0}\right)\right.$ implies that $x^{*} \in T\left(x^{*}\right)$.

Hence, the proof is complete.

\subsubsection{Application of Theorem 3.1.2, for applying it in the case of finite players}

The set of best-play is the strategy of sets by which we can get our expected optimum outcome easily. Consider, $P, Q$, and $R$ be the three players to a game. Let, $\bar{a}, \bar{b}$, and $\bar{c}$ be their probability distributions over the action sets. Also let $\alpha, \beta$, and $\gamma$ are their payoff functions. $A(\bar{b}, \bar{c})$ denotes the set of best-play $\bar{a}^{\prime}$ s. Then it is easy to see that $A(\bar{b}, \bar{c})$ is a convex closed set. $B(\bar{c}, \bar{a})$ denotes the set of best-play $\bar{b}^{\prime}$ s. Then it is easy to see that $B(\bar{c}, \bar{a})$ is a convex closed set. Similarly, $C(\bar{a}, \bar{b})$ is also a closed convex set. 
Define a function $T:\left(\begin{array}{l}\bar{a} \\ \bar{b} \\ \bar{c}\end{array}\right) \rightarrow\left(\begin{array}{c}A(\bar{b}, \bar{c}) \\ B(\bar{c}, \bar{a}) \\ C(\bar{a}, \bar{b})\end{array}\right)$. Then if we could apply theorem 3.1.1, we have $\left(\begin{array}{l}\bar{a} \\ \bar{b} \\ \bar{c}\end{array}\right) \in T\left(\begin{array}{l}\bar{a} \\ \bar{b} \\ \bar{c}\end{array}\right)$ that easily displays the existence of Nash equilibrium. The requirement we need now is to verify our set up those contents the conditions of Theorem 3.1.2. The first two conditions are satisfied trivially.

Now, the only requirement to show that $\{((x, y), T(x, y)\}$ is closed and for this we need to show $((x, y), T(x, y)) \rightarrow\left(x^{*}, y^{*}\right)$, where $x^{*}=\left(x_{0}, y_{0}\right)$ and $y^{*}=T\left(x_{0}, y_{0}\right)$.

$$
\begin{aligned}
& \text { Put, } x=(\bar{a}, \bar{b}, \bar{c}) \text { and } y=(\bar{p}, \bar{q}, \bar{r}) . \\
& \qquad \begin{aligned}
\left(x^{*}, y^{*}\right) \in T\left(x^{*}\right) \Leftrightarrow & y^{*} \in T\left(x^{*}\right) \\
\Leftrightarrow & \alpha\left(\overline{p^{*}}, \overline{b^{*}}, \overline{c^{*}}\right) \geq \alpha\left(\overline{a^{\prime}}, \overline{b^{*}}, \overline{c^{*}}\right) \text { for all } \overline{a^{\prime}}, \\
& \beta\left(\overline{a^{*}}, \overline{q^{*}}, \overline{c^{*}}\right) \geq \beta\left(\overline{a^{\prime}}, \overline{b^{*}}, \overline{c^{*}}\right) \text { for all } \overline{b^{\prime}},
\end{aligned}
\end{aligned}
$$

and

$$
\gamma\left(\overline{a^{*}}, \overline{b^{*}}, \overline{r^{*}}\right) \geq \gamma\left(\overline{a^{*}}, \overline{b^{*}}, \overline{c^{\prime}}\right) \text { for all } \overline{c^{\prime}}
$$

which shows that $\{x, T(x)\}$ is closed and hence we conclude that the above argument can easily apply to any finite players. 


\subsection{Application in the games for the area of quality management}

The questions arising from the analysis of the quality of a tangible or intangible product can be answered from the game theory in some cases. The creation of a tangible or intangible product depends on two groups of factors where one group raises the values of the product's quality and the other group lowers the values of the product's quality indicators. So, the first player is detected by the factors which raise the values of the quality and the other player is determined by the other group of factors. The former player "wishes" to make a high-quality product when the later "wishes" creating a poor-quality product. The outcome of the competition is the ultimate quality of the product.

From the devoted scientific notation, we denote by

$B_{1}=\left\{\beta_{1}, \beta_{2}, \ldots, \beta_{i}, \ldots, \beta_{m}\right\}$ the set of factors that results in the increase of the values of the quality of the indicators, and by

$B_{2}=\left\{b_{1}, b_{2}, \ldots, b_{j}, \ldots, b_{n}\right\}$ the set of factors that results in decrease. Each player has a certain impact on the values of the quality indicators at some point of time from the cycle of tangible or intangible product.

Each player is sure to choose an action $\beta_{i}$ from $B_{1}$ and $b_{j}$ from $B_{2}$. The actions refer to the influence of factor $i$ on the values of the quality indicators. The utility of choosing action $\beta_{i}$ by the first player can be explained mathematically by a real function and a win situation for the first player that proves the interpretation by its values. In this circumstance, function $f_{2}\left(\beta_{i}, b_{j}\right)$ represents the second player's loss. The fact that the sum of the game is null can be written as according to the specialty literature (Owen [16]):

$$
f_{1}\left(\beta_{i}, b_{j}\right)+f_{2}\left(\beta_{i}, b_{j}\right)=0 .
$$

In order to achieve maximum gain, how the first player should choose the action therefore remains the question, given that the other player has 
similar objective (the term utility was proposed by Von Neumann). The Nash equilibrium in its own strategy is showcased by strategic profile where the strategy of each player is also the best response to the other player's strategy. Therefore, the contrasting situations involving the creation of tangible or intangible products of a high quality level and their management can be given by a model with the use of amalgamation of the theories (see Figure 3.1):

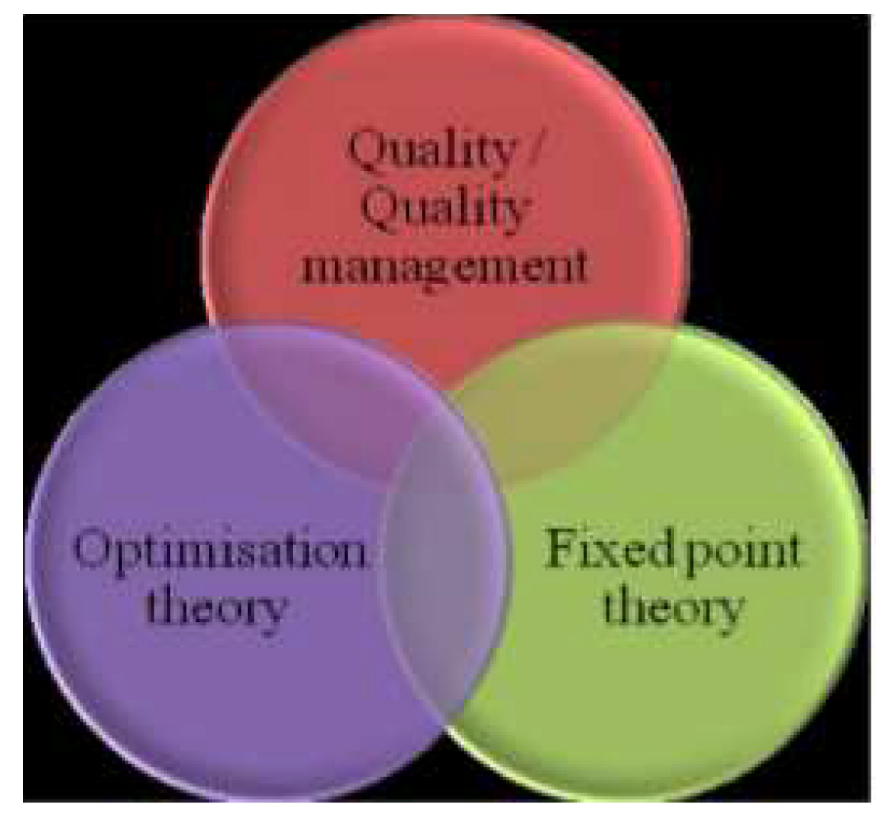

Figure 3.1. Representing the interconnection between the theories.

The fact that fixed point theory can be put to use to the optimization problems, game theory problems and problems arising from Nash equilibrium has been proved by many authors (i.e., Lin [11], Yang and Yu [23], Yu and Yang [24]).

Example 3.2.1. In order to increase the number of candidates that plans to file in their courses, the quality of the promotion services in universities $U_{1}$ and $U_{2}$, based on the data received from the analysis of the number of students that applied in the previous years, the marketing services of these universities came to the conclusions shown in the table below Table 1 and Table 2, respectively. 
Table 1. Marketing services of the universities $U_{1}$ and $U_{2}$

\begin{tabular}{|c|c|c|}
\hline $\begin{array}{c}U_{1} \text { represents the strategy } \\
\text { that is generated by its set } \\
B_{1}=\left\{\beta_{1}, \beta_{2}\right\} \text { of possible } \\
\text { actions }\end{array}$ & $\begin{array}{c}U_{2} \text { represents the } \\
\text { strategy that is generated } \\
\text { by its set } B_{2}=\left\{b_{1}, b_{2}\right\} \text { of } \\
\text { possible actions }\end{array}$ & $\begin{array}{l}\text { Result (that can change in the } \\
\text { percentage of candidates } \\
\text { which desire to enroll to the } \\
\text { university) }\end{array}$ \\
\hline $\begin{array}{c}\quad \beta_{1} \\
\text { (uses flyers) }\end{array}$ & $\begin{array}{c}\quad b_{1} \\
\text { (uses flyers) }\end{array}$ & $\begin{array}{c}f_{1}\left(\beta_{i}, b_{i}\right)=15\left(U_{1} \text { wins }\right) \\
f_{2}\left(\beta_{i}, b_{j}\right)=-15\left(U_{2} \text { loses }\right)\end{array}$ \\
\hline $\begin{array}{c}\quad \beta_{1} \\
\text { (uses flyers) }\end{array}$ & $\begin{array}{c}b_{1} \\
\text { (uses advertising media) }\end{array}$ & $\begin{array}{c}f_{1}\left(\beta_{i}, b_{j}\right)=11\left(U_{1} \text { wins }\right), \\
f_{2}\left(\beta_{i}, b_{j}\right)=-11\left(U_{2} \text { loses }\right)\end{array}$ \\
\hline $\begin{array}{c}\beta_{2} \\
\text { (uses advertising media) }\end{array}$ & $\begin{array}{c}b_{2} \\
\text { (uses flyers) }\end{array}$ & $\begin{array}{c}f_{1}\left(\beta_{i}, b_{j}\right)=-17\left(U_{1} \text { loses }\right) \\
f_{2}\left(\beta_{i}, b_{j}\right)=17\left(U_{2} \text { wins }\right)\end{array}$ \\
\hline $\begin{array}{c}\beta_{2} \\
\text { (uses advertising media) }\end{array}$ & $\begin{array}{c}b_{2} \\
\text { (uses advertising media) }\end{array}$ & $\begin{array}{c}f_{1}\left(\beta_{i}, b_{j}\right)=-7\left(U_{1} \text { loses }\right) \\
f_{2}\left(\beta_{i}, b_{j}\right)=7\left(U_{2} \text { wins }\right)\end{array}$ \\
\hline
\end{tabular}

Table 2. Summary of results

\begin{tabular}{|c|c|c|c|}
\hline & $b_{1}$ & $b_{2}$ & \\
\hline 1 & 15 & 11 & $\operatorname{Min}\{15,11\}=11$ \\
\hline 2 & -17 & 7 & $\operatorname{Min}\{-17,7\}=-17$ \\
\hline & $\operatorname{Max}\{15,-12\}=15$ & $\operatorname{Max}\{11,7\}=11$ & $\operatorname{Min}\{15,11\}=11=\operatorname{Max}\{11,-17\}$ \\
\hline
\end{tabular}

The game offers equilibrium point, $\left(\beta_{i}, b_{j}\right)$, that matches the result of the game. University $U_{1}$ eagerly uses action $\beta_{i}$, giving the notation to use the flyers. On the other hand, university $U_{2}$ tries to lessen loss because of selecting action $b_{j}$.

\subsection{Application in Cournot Oligopoly model}

Cournot Oligopoly [19] model is such a model for which the following assumption satisfies:

(i) The number of firms $(m)$ is finite. 
(ii) A firm $i$ is able to produce commodity $i$ for $i \in\{1,2, \ldots, m\}\left(\left(P_{i}\right)\right.$ and $\left(Q_{i} \geq 0\right)$ denote the price and the quantity of commodities, respectively).

(iii) All commodities are accurately divisible.

(iv) The aim of each firm is to select an amount of product that can be able to maximize its own gain given the levels of the production selected by other firms.

Suppose $Q_{-i}=\left(Q_{1}, Q_{2}, \ldots, Q_{i-1}, Q_{i+1}, \ldots, Q_{m}\right)$ be the quantities which is regarded as vector quantities produced by the other firms. We may consider that:

$$
P_{i}=p_{i}\left(Q_{i}, Q_{i-1}\right)=u_{i}-v_{i} Q_{i}+\sum_{j \neq i} d_{i j} Q_{j}, \quad i=1,2, \ldots, m,
$$

where, price $P_{i}$ is decrementing in its quantity $Q_{i}$ and, because of fulfilling between the commodities, is considered to be incrementing in the quantities $Q_{j}, j \neq i$, of the other firms (the parameters $u_{i}, v_{i}$, and $d_{i j}$ are taken as positive).

Each firm for $i \in\{1,2, \ldots, m\}$ has a function (known as cost function) which is linear written as $C_{i}\left(Q_{i}\right)=c_{i} Q_{i}$ with $u_{i}>c_{i}>0$. The profit $\alpha_{i}$ of firm $i \in\{1,2, \ldots, m\}$ is

$$
\alpha_{i}\left(Q_{i}, Q_{-i}\right)=Q_{i} p_{i}\left(Q_{i}, Q_{-i}\right)-c_{i} Q_{i}
$$

For each firm $i \in\{1,2, \ldots, m\}$ satisfies, if $\left(Q_{1}^{*}, Q_{2}^{*}, \ldots, Q_{m}^{*}\right) \in \mathbb{R}_{+}^{n}$ is a tuple of Cournot-Nash equilibrium:

$$
\alpha_{i}\left(Q_{i}^{*}, Q_{-i}^{*}\right) \geq \alpha_{i}\left(Q_{i}, Q_{-i}^{*}\right)
$$

for all $Q_{i} \in \mathbb{R}_{+}$. This equilibrium exists if $2 v_{i}>\sum_{j \neq i} d_{i j}$ for each firm $i \in\{1,2, \ldots, m\}$. 
When the only comment is such that all goods are exactly divisible is not satisfied, in such case the discrete Cournot-Nash equilibrium is explored. The goods that are produced and sold in integer quantities; they are, mobile phones, books, etc. Besides, numerous divisible goods, such as mangoes, vegetables, bananas etc. are sold in discrete quantities. For each firm $i \in\{1,2, \ldots, m\}$ satisfies, if $\left(Q_{1}^{*}, Q_{2}^{*}, \ldots, Q_{m}^{*}\right) \in \mathbb{Z}_{+}^{m}$ is a tuple of discrete Cournot-Nash equilibrium:

$$
\alpha_{i}\left(Q_{i}^{*}, Q_{-i}^{*}\right) \geq \alpha_{i}\left(Q_{i}, Q_{-i}^{*}\right)
$$

for all $Q_{i} \in \mathbb{Z}_{+}$.

A firm $i \in\{1,2, \ldots, m\}$ may maximize its gain $\alpha_{i}\left(Q_{i}, Q_{-i}\right)$ of its quantity which is regarded as optimal integer given by the retraction function:

$$
r_{i}\left(Q_{-i}\right)=\left[\frac{u_{i}-c_{i}}{2 v_{i}}+\sum_{j \neq i} \frac{d_{i j}}{2 v_{i}} Q_{j}\right]
$$

Retraction function helps in explaining the quantity choice of the firms to how one firm reacts to the quantity choice of the other firm. In this case, here we use the symbol $[h]$ which means the greatest nearest integer to $h$ and for the values of $i \in\{1,2, \ldots, m\}$ satisfies $r_{i}\left(Q_{-i}\right) \geq 0$ for every $Q \in \mathbb{Z}_{+}^{m}$ (because $u_{i}>c_{i}>0$ ).

Define the function $F: \mathbb{Z}_{+}^{m} \rightarrow \mathbb{Z}^{m}$ by

$$
F_{i}\left(Q_{i}, Q_{-i}\right)=r_{i}\left(Q_{-i}\right)-Q_{i}, i=1,2,3, \ldots, m .
$$

A discrete zero point of $F$ is a discrete Cournot-Nash equilibrium. Then clearly, if $2 v_{i}>\sum_{j \neq i} d_{i j}, i=1,2,3, \ldots, m$. Brouwer's fixed point theorem can show that function $F$ will have a discrete zero point. Thus, the final assumption shows that the Cournot Oligopoly model in such cases will have a discrete Cournot-Nash equilibrium when $2 v_{i}>\sum_{j \neq i} d_{i j}, i=1,2,3, \ldots, m$. 


\section{Conclusion}

As analysis in fixed point theory has notably elongated in recent few years which is actually tremendous in the field of both pure and applied mathematics. We have garnished this paper, in such a way that from start to finish it clarify the sense how Kakutani's fixed point theorem have been worked in different fields of game theory with their mathematical explanations. We have discussed the three applications (like as; Nash equilibrium, the Games for the area of quality management, and Cournot Oligopoly model) of this theorem in the field of game theory. Finally, we are strongly optimistic that the future researchers will be developed more to apply this theorem following the idea about our proposed applications in this article that must create a remarkable influence on science, and engineering fields.

\section{References}

[1] K. J. Arrow and G. Debreu, Existence of an equilibrium for a competitive economy, Econometrica: Journal of the Econometric Society 22(3) (1954), 265-290.

DOI: https://doi.org/10.2307/1907353

[2] K. C. Border, Fixed Point Theorems with Applications to Economics and Game Theory, Cambridge University Press, 1985.

$$
\text { DOI: https://doi.org/10.1017/CBO9780511625756 }
$$

[3] L. E. J. Brouwer, Über abbildung von mannigfaltigkeiten, Mathematische Annalen 71(1) (1911), 97-115.

DOI: https://doi.org/10.1007/BF01456931

[4] C. Buxton, Brouwer Fixed-Point Theorem, 2016.

[5] S. Cobzaş, Fixed point theorems in locally convex spaces: The Schauder mapping method, Fixed Point Theory and Applications 1 (2006); Article 57950.

DOI: https://doi.org/10.1155/FPTA/2006/57950

[6] S. Eilenberg and D. Montgomery, Fixed point theorems for multi-valued transformations, American Journal of Mathematics 68(2) (1946), 214-222.

DOI: https://doi.org/10.2307/2371832 
[7] K. Fan, Fixed-point and minimax theorems in locally convex topological linear spaces, Proceedings of the National Academy of Sciences of the United States of America 38(2) (1952), 121-126.

DOI: https://doi.org/10.1073/pnas.38.2.121

[8] I. L. Glicksberg, A further generalization of the Kakutani fixed point theorem, with application to Nash equilibrium points, Proceedings of the American Mathematical Society 3(1) (1952), 170-174.

DOI: https://doi.org/10.2307/2032478

[9] S. Kakutani, A generalization of Brouwer's fixed point theorem, Duke Mathematical Journal 8(3) (1941), 457-459.

DOI: https://doi.org/10.1215/S0012-7094-41-00838-4

[10] R. Kannan, Some results on fixed points, Bulletin Calcutta Mathematical Society 60 (1968), 71-76.

[11] Z. Lin, Essential components of the set of weakly Pareto-Nash equilibrium points for multiobjective generalized games in two different topological spaces, Journal of Optimization Theory and Applications 124(2) (2005), 387-405.

DOI: https://doi.org/10.1007/s10957-004-0942-0

[12] J. F. Nash, Equilibrium points in $n$-person games, Proceedings of the National Academy of Sciences 36(1) (1950), 48-49.

DOI: https://doi.org/10.1073/pnas.36.1.48

[13] J. F. Nash, Non-cooperative Games, Ph.D. Thesis, Mathematics Department, Princeton University, 1950b.

[14] J. Nash, Non-cooperative games, Annals of Mathematics 54(2) (1951), 286-295.

DOI: https://doi.org/10.2307/1969529

[15] J. Von Neumann, Zur theorie der gesellschaftsspiele, Mathematische Annalen 100(1) (1928), 295-320.

DOI: https://doi.org/10.1007/BF01448847

[16] G. Owen, Teoria Jocurilor, Bucureşti, Romania: Editura Tehnică, 1974.

[17] A. Rubinstein, Experience from a course in game theory: Pre- and postclass problem sets as a didactic device, Games and Economic Behavior 28(1) (1999), 155-170.

DOI: https://doi.org/10.1006/game.1999.0723

[18] H. Scarf, The approximation of fixed points of a continuous mapping, SIAM Journal on Applied Mathematics 15(5) (1967), 1328-1343.

DOI: https://doi.org/10.1137/0115116 
[19] G. van der Laan, A. J. J. Talman and Z. Yang, Combinatorial integer labeling theorems on finite sets with applications, Journal of Optimization Theory and Applications 144(2) (2010), 391-407.

DOI: https://doi.org/10.1007/s10957-009-9603-7

[20] R. V. Vohra, Advanced Mathematical Economics, Routledge, 2004.

DOI: https://doi.org/10.4324/9780203799956

[21] J. Von Neumann, Uber ein okonomisches gleichungssystem und eine verallgemeinerung des brouwerschen fixpunktsatzes, In Ergebnisse eines Mathematischen Kolloquiums 8 (1937), 73-83.

[22] J. Von Neumann, O. Morgenstern and H. W. Kuhn, Theory of Games and Economic Behavior (Commemorative Edition), Princeton University Press, 2007.

[23] H. Yang and J. Yu, Essential components of the set of weakly Pareto-Nash equilibrium points, Applied Mathematics Letters 15(5) (2002), 553-560.

DOI: https://doi.org/10.1016/S0893-9659(02)80006-4

[24] J. Yu and H. Yang, The essential components of the set of equilibrium points for set-valued maps, Journal of Mathematical Analysis and Applications 300(2) (2004), 334-342.

DOI: https://doi.org/10.1016/j.jmaa.2004.06.042 